\title{
Modeling and Benchmarking Ultra-Wideband Localization for Mobile Robots
}

Alexander Bahr, Alexander Feldman, James Colli-Vignarelli, Stephan Robert, Catherine Dehollain and Alcherio Martinoli

\begin{abstract}
Ultra-Wideband Impulse Radio (UWB-IR) is a technology that has great potential to solve numerous mobile robotic and asset tracking problems in GPS-denied environments. Our goal is to help software and hardware designers in improving the state-of-the-art in UWB-based robotic localization. We developed a test-bed where an UWB transmitter is attached to a mobile robot. By combining the received signals with the robot's position log acquired through the dead-reckoning sensors, we obtain UWB signals which are well referenced with respect to the transmitter-receiver distance and orientation. In addition, we provide a model for every component of the setup. The entire setup allows us to simulate from first principles every aspect of an UWB localization system and then to implement low-level signal processing as well as higher-level modulation and localization techniques. We implement an Automatic Gain Control (AGC) algorithm to demonstrate the rapid proto-typing capabilities of the test-bed. Our work shows how an UWB robotic system and its models can be involved in all phases of the development of a technology that can help robot's navigation, localization and communication algorithms.
\end{abstract}

\section{INTRODUCTION}

UWB localization offers many advantages compared to other methods for localization and asset tracking in GPSdenied environments. Localization technologies based on laser [1] require a line of sight between the beacon and the asset and ultrasound-based ones such as the CRICKET [2] experience a serious degradation in performance in Non-Lineof-Sight (NLoS) conditions. UWB pulses, on the other hand, are resilient to frequency dependent absorption due to their large bandwidth.

The purpose of the UWB test-bed is to improve the stateof-the-art in localization algorithms. Theoretical estimations for practical systems indicate, that accuracies of $2 \mathrm{~cm}$ or less can be achieved under ideal conditions [3], but accuracies of $10-15 \mathrm{~cm}$ are more typical for Line-of-Sight $(\operatorname{LoS})$ and $30-50 \mathrm{~cm}$ for NLoS applications [4]. Reducing the localization error to values closer to the theoretical limit, particularly in NLoS scenarios, requires a comprehensive approach which

The work presented in this paper was supported by the National Competence Center in Research on Mobile Information and Communication Systems (NCCR-MICS), a center supported by the Swiss National Science Foundation under grant number 51NF40-111400.

Alexander Bahr and Alcherio Martinoli are with the Distributed Intelligent Systems and Algorithms Laboratory, Ecole Polytechnique Fédérale de Lausanne (EPFL).firstname. lastnamedepfl. ch

Alexander Feldman and Stephan Robert are with the Institute for Information and Communication Technologies, Haute Ecole d'Ingénierie et de Gestion du Canton de Vaud (HEIG-VD).firstname. lastname@heig-vd. ch

James Colli-Vignarelli and Catherine Dehollain are with the Radio Frequency Integrated Circuit Group, Ecole Polytechnique Fédérale de Lausanne (EPFL).firstname.lastnamedepfl.ch includes the modeling of the individual blocks of the system and benchmarking on real hardware.

Deploying and modifying of robotic test-beds and, in particular, UWB localization systems, is typically expensive. To reduce this cost we use model-based methods where we first build part of the test-bed, then model the whole system and finally use the model to optimize the performance of the test-bed. To create the necessary models we use various techniques such as modeling from first-principles, numerics, approximation methods, and importance sampling. The result is a comprehensive model that combines slow processes such as the movement of a robot (seconds) and fast processes such as the transmission of localization signals (parts of nanoseconds).

In order to verify the fidelity of our model we need to compare its output to measurements from the real hardware. As the localization accuracy of an UWB system depends on several factors, such as Signal-to-Noise Ratio (SNR) of the received signal, the bandwidth of the UWB pulse, and the geometry between the transmitter and the receiver, it is important that the collection of the signals is synchronized with a log of all parameters affecting the signal. Using a transmitter attached to a robot has several advantages over static setups. First, it allows us to combine logs of the robot pose $(x, y$ position and orientation $\theta$ ) with the acquired signal. We can track the pose with high accuracy using an external camerabased system, odometry or a combination of both. Second, we can pre-program a path in order to vary the surroundings while maintaining the same transmitter trajectory. In addition our setup provides a MATLAB interface to the receiver which allows rapid prototyping for higher-level signal processing in near-real-time.

The contributions of the paper are the following. (1) We present a robotic UWB-IR test-bed. (2) We provide a comprehensive model of the test-bed. (3) We use this test-bed to create a benchmark of wave forms that can be compared with the model output and used for design, development, and integration of UWB-IR robotic systems. (4) With the help of a robot we validate this benchmark. (5) We illustrate the usability of the entire system by presenting a user case: the development of Automated Gain Control (AGC) for increasing the performance of signal processing algorithms in a multipath environment.

\section{RELATED WORK}

UWB localization is discussed in the context of indoor robot navigation in [6]. We extend this paper by providing a 
systematic UWB data-set where the signal is collected in realworld conditions and is sampled over multiple trajectories. Our work can help such efforts by reducing or eliminating the need to deploy a fully functional commercial UWB localization which, at the time of the writing of this paper, still require substantial investment. UWB literature [7], [8] reports on various experiments in office environments, however, these are static, and only the summary has been made public.

Empirical data for UWB localization in several environments as well as a statistical model are provided in [9]. The authors of [10] evaluate the performance of their robotic system and report UWB localization accuracy close to $20 \mathrm{~cm}$.

\section{TEST-BED}

The test-bed U-LITE developed at EPFL consists of a transmitter which is attached to a KHEPERA III, a small (diameter $=12 \mathrm{~cm}$ ) mobile robot [11] (Fig. 1). The transmitter generates UWB pulses with a variable Pulse Repetition Rate of up to $10 \mathrm{MHz}$ which are centered around $4.25 \mathrm{GHz}$ with a bandwidth of at least $500 \mathrm{MHz}$ [12].

The design of the transmitter and receiver antennas is described in [13].

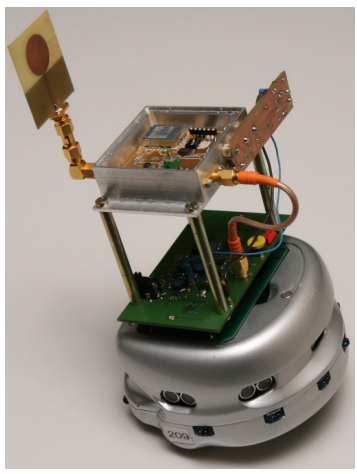

Fig. 1: The mobile transmitter consisting of a KHEPERA III robot, the modulator (green circuit board), the UWB transmitter and the antenna

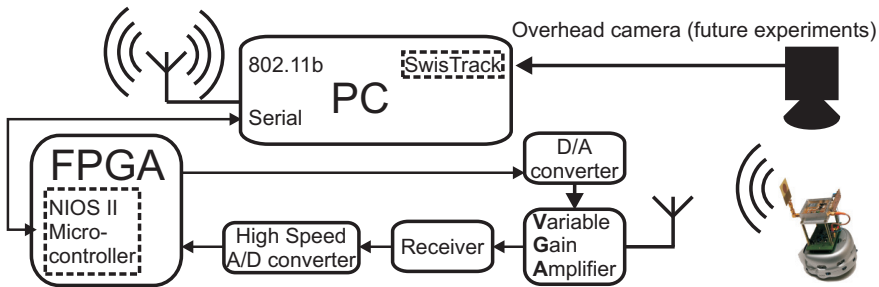

Fig. 2: The test-bed. Transmitter: KHEPERA III robot with UWB modulator and transmitter. Receiver: analog front end, ADC and FPGA for ADC interfacing and low-level processing, embedded NIOS II soft core. PC: for data logging and high-level signal processing, robot tracking and robot control

The receiver consists of two Low-Noise Amplifiers (LNAs) with voltage controlled gain [14]. The analog signal is directly fed into an Analog-to-Digital converter (ADC) $(2.816 \mathrm{GHz}$ sampling frequency and 8 bit resolution). Attached to the ADC is a Field-Programmable Gate Array (FPGA). The FPGA is used for low level real-time signal processing such as sample and store, threshold and maximum detection [15].

We implemented a MATLAB library which controls communication with the UWB receivers and allows us to use the MATLAB environment to prototype complex signal processing algorithms. While the serial transfer introduces an additional delay over signal processing within the FPGA, the overall system is still fast enough for control loops running at $\approx 10 \mathrm{~Hz}$. To obtain the robot position we relied on the dead-reckoned position of the robot, which after some careful calibration as described in [11], is accurate to within $\approx 1 \%$ of the distance traveled which is enough for the short distances within the following experiments.

A detailed description of the entire test-bed can be found in [16].

\section{NumericAl Model}

In what follows we describe a numerical Simulink ${ }^{\circledR}$ model of the robotic UWB test-bed. The model comprises several components and we use the acronym ELISSA (EPFL robotIc localization teSt-bed Simulator) to refer to it. We have used this model to generate a synthetic UWB benchmark. In creating the numerical model we have used first-principles where possible, i.e., we have created a compositional model. We have used experimental measurements to validate and calibrate the model. The advantage of the numerical model is that it can be customized to a large variety of UWB test-beds.

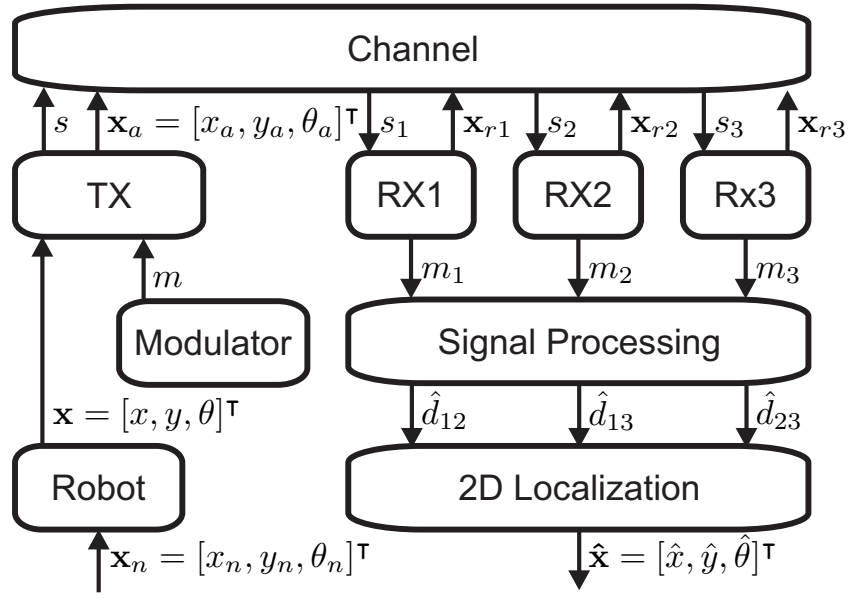

Fig. 3: Overview of ElisSA

Figure 3 shows the individual building blocks of the model. The robot is given a trajectory in the form of a series of poses $\mathbf{x}_{n}$. As it moves, the time-dependent pose of the antenna of the UWB transmitter attached to the robot is $\mathbf{x}_{a}$. The emitted signal $\mathrm{s}$ is modulated by a pulse train $\mathrm{m}$. The channel model simulates the signal attenuation due to spreading loss. The signals $s_{1} \ldots s_{3}$ reach the respective receivers at positions $\mathbf{x}_{r 1} \ldots \mathbf{x}_{r 3}$ and each receiver provides the demodulated signal $m_{1} \ldots m_{3}$. The signal processing stage obtains the time of arrival of each UWB-pulse through matched filtering or simple thresholding and computes a time difference of 

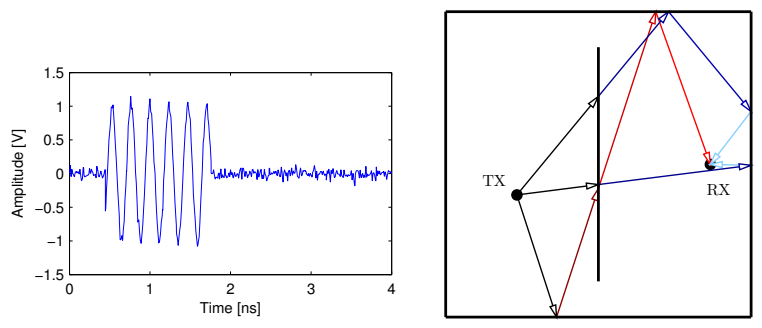

Fig. 4: Simulation of the RF Fig. 5: Output of ray-tracing transmitter output signal as used to compute the pulse components

arrival $\Delta \mathrm{t}_{1} \ldots \Delta \mathrm{t}_{3}$ with respect to a common synchronization signal. It then computes the pairwise differences $\hat{\mathrm{d}}_{12}, \hat{\mathrm{d}}_{13}, \hat{\mathrm{d}}_{23}$ between these time stamps which constrain the position of the robot to the intersection of a set of hyperbolae. From these pairwise time differences the localization block estimates the position of the robot $\hat{\mathbf{x}}$. The following section describes the individual building blocks of the model.

\section{A. Robot}

The robot used in the test-bed (Fig. 1) uses a differential drive mechanism (wheel distance $b$ ). The input to its model are the pose $\mathbf{x}_{r}(\mathrm{t}-1)=\left[x_{r}(\mathrm{t}-1), y_{r}(\mathrm{t}-1), \theta_{r}(\mathrm{t}-1)\right]$, the speed $v_{l}$ of the left wheel and $v_{r}$ of the right wheel. The output of the model is the robot's pose $\mathbf{x}_{r}(\mathrm{t})$ at time $t$ which can directly be transformed into the pose of the transmitting antenna $\mathbf{x}_{\mathrm{a}}(\mathrm{t})$. The incremental pose change is computed as outlined in [17]. The model accounts for wheel slip as proposed in [18]

\section{B. Modulator and RF Transmitter}

The purpose of the analog RF transmitter [12] is to generate a signal of very short duration $(2-3 \mathrm{~ns})$. This is achieved by mixing the signal generated by a fast oscillator $(4.25 \mathrm{GHz})$ with a trapezoidal signal that acts as a fast switch. To generate this driving signal, the analog RF transmitter "squares" the modulator output by using a fast comparator (NC7S14) and then uses the output of this comparator to drive (discharge/charge) a variable capacitor $(41-96 \mathrm{pF})$. We have modeled this as:

$$
V_{c}(t)=V_{\mathrm{IN}}\left[1-e^{-t / R C}\right]
$$

where $V_{c}(t)$ is the output voltage, $V_{\mathrm{IN}}$ is the initial voltage, $R$ is the resistance and $C$ is the capacitance of the variable capacitor. The signal measured after the capacitor consists of two exponents which are converted to a rectangular pulse by a second comparator. To offset the central frequency of the transmitted pulse it is mixed with a $4.25 \mathrm{GHz}$ sine wave. The resulting signal is shown in Fig. 4.

\section{Antennas and Transmission Channel}

Channel and antenna models are topics of their own and we provide only a brief description due to page limitations. At high frequencies electromagnetic propagation can be approximated with methods from optics and we use 2-D raytracing [19] as an approximation for the channel model. This ray-tracing model was implemented as a stand-alone C library. Figure 5 illustrates our approach.

Figure 5 illustrates signal propagation in a room with an obstacle (wall) between the transmitter and the receiver. In this example, we have imposed a limit of four reflections or absorptions for the ray-tracing algorithm. By using this algorithm we can compute the delays between the reflections of the transmitted impulse. 2-D modeling is sufficient for the creation of NLoS signal benchmark, and, for other applications of ELISSA, it is trivial to perform 3-D ray-tracing at increased computational cost.

\section{RF Receiver}

COTS oscillators such as the ones used in our UWB transmitter and receiver produce slightly different waveforms due to manufacturing tolerances and environmental factors. Such differences negatively affect the received signal, increase the Signal-to-Noise Ratio (SNR), and subsequently increase the Bit Error Rate (BER) in communication applications or decrease the accuracy in localization applications. We have used numerical simulation to simulate an I/Q demodulationbased receiver. Its Simulink ${ }^{\circledR}$ model is shown in [16]. The input signal is a pulse wave with a period cycle of $1 \%$. It is mixed with a sine wave with a final frequency of $4.25 \mathrm{GHz}$. On the receiver side, the signal is down-converted by using $4.20 \mathrm{GHz}$ sine and cosine waves. The resulting in-phase and quadrature parts of the signal are then squared and summed to produce the final received signal. The output of the quadrature generator is tapped to simulate the work of a traditional, nonI/Q-based receiver.

\section{E. Signal Processing}

We have implemented threshold (leading edge) pulse detection. A pulse is detected when $V>0.6 \mathrm{~V}$ after which the detection is paused for $0.5 \mu$ s. After detecting the positions of 10 pulses we computed the Mean Square Error (MSE) from the transmission times. The I/Q demodulated design we propose has an MSE of $1.44 \mathrm{~ns}$ that translates to a range error of $0.43 \mathrm{~m}$ (the TDoA localization MSE is going to be smaller due to the averaging of several samples).

\section{F. $2 D$ Localization}

To validate our simulation results we solve the well-known 2D multilateration problem [20], i.e., we try to minimize 2 thus solving for the robot position $\hat{\mathbf{x}}=[x+\tilde{x}, y+\tilde{y}]^{\top}$ with $\tilde{x}$ and $\tilde{y}$ representing the error in the position estimate.

$$
\begin{aligned}
f(\hat{x}, \hat{y})= & {\left[\hat{d}\left(\mathbf{x}_{r 1}\right)-\hat{d}\left(\mathbf{x}_{r 2}\right)-\frac{\hat{d}_{12}}{\mathrm{c}}\right]^{2}+} \\
& {\left[\hat{d}\left(\mathbf{x}_{r 1}\right)-\hat{d}\left(\mathbf{x}_{r 3}\right)-\frac{\hat{d}_{13}}{\mathrm{c}}\right]^{2}+} \\
& {\left[\hat{d}\left(\mathbf{x}_{r 2}\right)-\hat{d}\left(\mathbf{x}_{r 3}\right)-\frac{\hat{d}_{23}}{\mathrm{c}}\right]^{2} } \\
\hat{d}\left(\mathbf{x}_{r n}\right)= & \sqrt{\left(\hat{x}-x_{r n}\right)^{2}+\left(\hat{y}-y_{r n}\right)^{2}}
\end{aligned}
$$

In (2), $\mathbf{x}_{r 1}, \mathbf{x}_{r 2}, \mathbf{x}_{r 3}$ represent the error-free positions of the three receivers. $\hat{d}_{12}, \hat{d}_{13}$, and $\hat{d}_{23}$ denote the Time Difference 


\begin{tabular}{lll}
\hline Mission & Description & $\begin{array}{l}\text { Approximate } \\
\text { Duration [s] }\end{array}$ \\
\hline 5 & $\begin{array}{l}\text { Robot is moving in a circular trajectory with } \\
\text { an absorber (red) in the middle of the table } \\
\text { Same as mission 5, but with two additional } \\
\text { reflectors in the corner }\end{array}$ & 243 \\
6 & 243 \\
\hline
\end{tabular}

TABLE I: Mission descriptions

of Arrival (TDoA) of the three pulses with $\hat{d}=d+\tilde{d}$ where $d$ denotes the true TDoA and $\tilde{d}$ the measurement noise. $\mathrm{c}$ denotes the speed of light.

We solve the multilateration problem with an algorithm where we iteratively split the arena in 4 quadrants and compute (2) for each of the midpoints. We then take the one which results in the smallest residual and iterate again.

We have discussed all building blocks of ELISSA. The input of this model is the speed of the differential drive motors and the output is the position as estimated by the UWB localization system.

\section{EXPERIMENTS}

We continue with a description of the experiments we have carried-out on U-LITE.

\section{A. Experimental Setup}

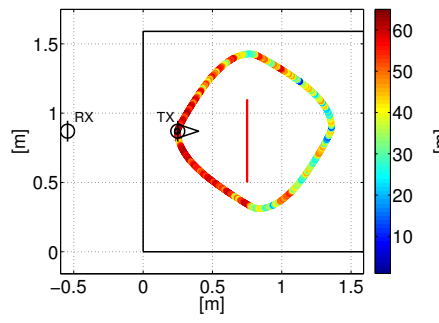

(a) Mission 5

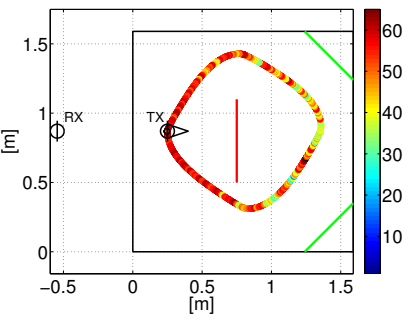

(b) Mission 6
Fig. 6: Mission 5 and 6: arena (black: arena outline, green: reflectors, red: absorber) with receiver and initial robot+transmitter position; vehicle tracks, the overlay is showing the average RSS

During all experiments the robot and the attached transmitter moved within a plane $160 \mathrm{~cm}$ by $160 \mathrm{~cm}$ arena. The transmitter antenna's center point was $20 \mathrm{~cm}$ above the arena and the antenna plane was perpendicular to the robot's heading vector as shown in Fig. 1. The position of the receiver antenna is shown in all plots in Fig. 6. For all mission logs the robot's start position represents the origin and heading $0 \mathrm{rad}$ is along the (horizontal) $\mathrm{x}$-axis. The missions are described in Table I and the descriptions for the remaining missions along with the associated data can be found at ftp://ultrawide.org/pub/ICUWB2012/.

Each run ended at the robot's starting position and we determined the accumulated navigation drift by measuring the distance between the final and the start position. The average drift was $\approx 2 \mathrm{~cm}$ with no value larger than $3 \mathrm{~cm}$.
To obtain geo-referenced UWB data sets, the logs on the PC which contained the UWB data were merged with the logs on the KHEPERA III robot which contained the robot pose. To ensure synchronization, both logs provided time-stamped data. As the robot can control the UWB transmitter, the beginning and the end of the robot's log were synchronized with the starting and stopping of the transmitter which can be easily detected in the UWB logs.

\section{B. UWB Wave Form Snapshots}

In our first experiment we recorded snapshots of the raw UWB signal. Streaming the entire sampled signal would generate 2.816 GBytes/s of data. We acquired $32 \mathrm{k}$ samplesnapshots from the FPGA's buffer which corresponds to a time window of $\approx 11 \mu$ s containing $\approx 110$ pulses. The snapshots were taken at a rate of $10 \mathrm{~Hz}$ and each sample was timestamped using the UWB receiver's internal counter. Fig. 8 shows two examples of the raw signal. Fig. 8a shows a single pulse and Fig. 8b a single pulse and its reflection.

The data collected during these eight missions is very useful as it allows UWB developers to design and test-their signal acquisition, localization and communication algorithms in a high-level environment such as MATLAB, compared to directly implementing them on the FPGA. The U-LITE single-receiver single-transmitter wave-form dataset as well as other data is available for download from ftp://ultrawide.org/pub/ICUWB2012/

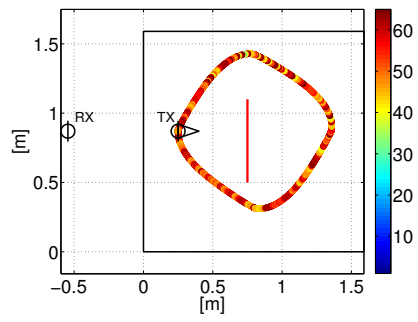

(a) Mission 5 with AGC

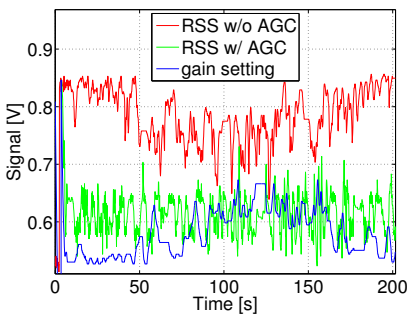

(b) RSS for mission 5 with and without AGC, gain setting
Fig. 7: Mission 5 carried out with AGC enabled

\section{Received Signal Strength (RSS)}

To obtain an understanding of the qualitative influence of the robot's pose and the influence of the obstacles we measured the RSS along all trajectories. Throughout all missions the receiver gain was not changed which allows us to clearly see the effect of obstacles and reflectors in the RSS. In mission 5 (Fig. 6a) we have an NLOS segment where the signals are almost completely blocked by the obstacle. Thus the signals in mission 6 (Fig. 6b) must be predominantly reflections during the NLOS part. The results for mission 1,2,3,4,7 and 8 were not plotted, but are available for download.

\section{Automatic Gain Control (AGC)}

The limited size of the arena allows us to collect most UWB pulses without adjusting the gain. For larger operating areas 


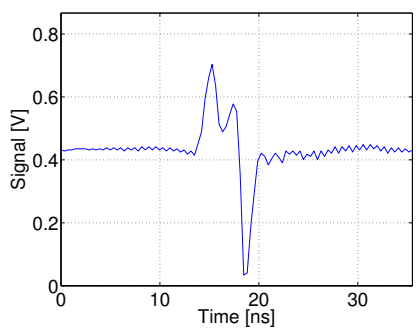

(a) Single UWB pulse

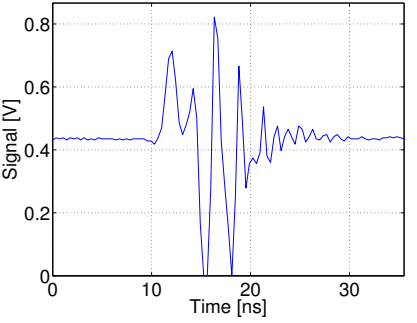

(b) UWB pulse with reflection
Fig. 8: Raw UWB signals

however which are encountered in practical applications and in cluttered environments with predominantly NLOS conditions such as in Fig. 6a and Fig. 6b the small dynamic range of very fast $\mathrm{ADCs}$ requires $\mathrm{AGC}$ in the analog receiver chain.

The rapid prototyping capabilities of our test-bed are particularly well suited to develop an AGC algorithm. The output of the control loop is connected to the Variable Gain Amplifier (VGA) in the analog receiver chain. The AGC algorithm itself can be implemented at different levels. For early prototypes of the AGC algorithm snap shots of the UWB signal were captured directly in MATLAB on the PC and MATLAB was used to determine the RSS from the snap shot, compute the required gain and set it. The frequency of the control loop was limited by the speed of the serial connection transmitting entire snapshots of raw UWB data and in order to speed up the control loop the second iteration of the algorithm relied on the RSS values computed by the FPGA. With this second iteration we obtained the results presented in Fig. 7. The control loop consisted of a simple proportional $\left(P_{\text {gain }}=0.1\right)$ control running at $10 \mathrm{~Hz}$.

We show the effect of the AGC in mission 5. When comparing Fig. 6a with Fig. 7a we notice how the RSS varies less throughout the robot track. Fig. 7b shows the RSS for mission 5 with and without AGC as well as the gain setting of the VGA over time. The standard deviation for the RSS without AGC is $\sigma_{n o A G C}=0.053$ and with AGC $\sigma_{A G C}=0.036$. Note that in order to best utilize the dynamic range of the $\mathrm{ADC}$, the $\mathrm{AGC}$ algorithm tries to maintain an average signal level closer to the midpoint $\left(V_{\text {target }}=0.61 \mathrm{~V}\right)$ which is lower than the average RSS in the runs without AGC.

\section{CONClusions AND Future WORK}

We present a test-bed for UWB localization where the transmitter is attached to a mobile robot. As this test-bed is used to improve the localization accuracy beyond the performance of existing commercial systems we need to develop an in-depth understanding of the noise sources which cumulate in the final localization error. As the entire test-bed has been developed by us we have complete insight into the hardware as well as the firmware. This allows us to model all building blocks of the entire test-bed which we present in this paper. We demonstrate the ability of our test-bed to get from initial simulations to working code via rapid-prototyping by implementing AGC on our receiver. The building blocks of the simulation are made available as well as the captured and geo-referenced UWB signals. Obtaining such data is usually difficult because of the prohibitive cost of acquiring such a system or because the closed hardware/software of commercial products does not provide access to non-processed data. At present the experiments have been performed using singlerobot (transmitter)/single-receiver setups. In the future we intend to extend our data-set to include multi-robot/multireceiver experiments.

\section{REFERENCES}

[1] A. Nasipuri and R. el Najjar, "Experimental evaluation of an angle based indoor localization system," in Proc. 4th Int Modeling and Optimization in Mobile, Ad Hoc and Wireless Networks Symp, 2006, pp. 1-9.

[2] N. B. Priyantha, A. K. Miu, H. Balakrishnan, and S. Teller, "The cricket compass for context-aware mobile applications," in 7th ACM MOBICOM, Rome, Italy, July 2001

[3] Z. Sahinoglu, S. Gezici, and I. Güvenc, Ultra-wideband Positioning Systems: Theoretical Limits, Ranging Algorithms, and Protocols, illustrated edition ed. Cambridge University Press, Oct. 2008.

[4] Ubisense. (2011, March) Ubisense data sheet. www . ubisense. net.

[5] N. Muscettola, P. P. Nayak, B. Pell, and B. C. Williams, "Remote agent: To boldly go where no AI system has gone before." Artificial Intelligence, vol. 103, no. 1-2, pp. 5-47, 1998.

[6] S. Krishnan, P. Sharma, Z. Guoping, and O. H. Woon, "A UWB based localization system for indoor robot navigation," in Proc. ICUWB'07, 2007, pp. 77-82.

[7] P. Pagani and P. Pajusco, "Experimental assessment of the UWB channel variability in a dynamic indoor environment," in Proc. of the 15th IEEE International Symposium on Personal, Indoor and Mobile Radio Communications (PIMRC) 2004, vol. 4, 2004, pp. 2973-2977.

[8] J. Cramer, R. Scholtz, and M. Win, "On the analysis of UWB communication channels," in Military Communications Conference Proceedings, 1999. MILCOM 1999. IEEE, vol. 2, 1999, pp. 1191-1195.

[9] N. Alsindi, B. Alavi, and K. Pahlavan, "Measurement and modeling of ultrawideband TOA-based ranging in indoor multipath environments," IEEE Transactions on Vehicular Technology, vol. 58, no. 3, pp. 10461058, March 2009.

[10] M. Segura, H. Hashemi, C. Sisterna, and V. Mut, "Experimental demonstration of self-localized ultra wideband indoor mobile robot navigation system," in Proc. of the 2010 International Conference on Indoor Positioning and Indoor Navigation (IPIN), September 2010, pp. 1-9.

[11] A. Prorok, A. Arfire, A. Bahr, J. R. Farserotu, and A. Martinoli, "Indoor navigation research with the Khepera III mobile robot: An experimental baseline with a case-study on ultra-wideband positioning," in Proc. of the 2010 Int. Conf. on Indoor Positioning and Indoor Navigation, Zurich, Switzerland, 2010, pp. 1-9.

[12] J. Colli-Vignarelli and C. Dehollain, "A discrete-components impulseradio ultra-wide band (IR-UWB) transmitter,' IEEE Trans. on Microwave Theory and Techniques, vol. 59, no. 4, pp. 1141-1146, 2011.

[13] G. Q. D. de Leon, J.-F. Zürcher, and A. Skrivervik, "Omnidirectional pulse dispersion of planar circular monopoles," in Proc. UWB'09, 2009, pp. 395- 399.

[14] J. Colli-Vignarelli and C. Dehollain, "A discrete-components impulseradio UWB Low-Noise Amplifier with voltage controlled-gain," in Proc. PRIME'10, July 2010, pp. 1-4.

[15] R. Merz, F. Chastellain, A. Blatter, C. Botteron, and P.-A. Farine, "An experimental platform for an indoor location and tracking system," in Proc. of the 2008 European Navigation Conference (ENC-GNSS 08), 2008.

[16] A. Feldman, A. Bahr, J. Colli-Vignarelli, S. Robert, C. Dehollain, and A. Martinoli, "Toward the deployment of an ultra-wideband localization test bed," in Proc. of the 74th Vehicular Technology Conference, San Frncisco, CA, USA, September 2011.

[17] R. Siegwart and I. R. Nourbakhsh, Introduction to Autonomous Mobile Robots. Bradford Company Scituate, 2004.

[18] S. Thrun, W. Burgard, and D. Fox, Probabilistic Robotics. Cambridge, MA, USA: The MIT Press, 2005.

[19] J. McKown and R. H. Jr., "Ray tracing as a design tool for radio networks," Network, IEEE, vol. 5, no. 6, pp. 27-30, nov 1991. 
[20] H. Lee, "Accuracy limitations of hyperbolic multilateration systems," Aerospace and Electronic Systems, IEEE Transactions on, vol. AES-11, no. 1, pp. 16-29, January 1975. 